\title{
Weight Optimization of Bed for Heavy Duty CNC Lathe Machine MGX200 using FEM
}

\author{
Haresh Modhavadiya, Rakesh Prajapati, Bhupesh Goyal
}

\begin{abstract}
In this paper, MGX200 CNC Lathe bed selected for the complete static and vibration analysis. The examination work completed to lessen the heaviness of bed without break its basic inflexibility and the precision of the machine instrument by expelling material.In this work, 3D CAD model done by using Autodesk Inventor Professional 2018 Software and analysis carried out in ANSYS 16.2 Software. The outcomes appeared with the assistance of qualities break down the impact of weight decrease on the basic trustworthiness of the machine bed when the weight decrease then decisions about the enhanced structure.
\end{abstract}

Keywords: Optimization, CNC Bed, FEM

\section{INTRODUCTION}

A CNC machine used for remove the material and give the desire shape and size as we required.

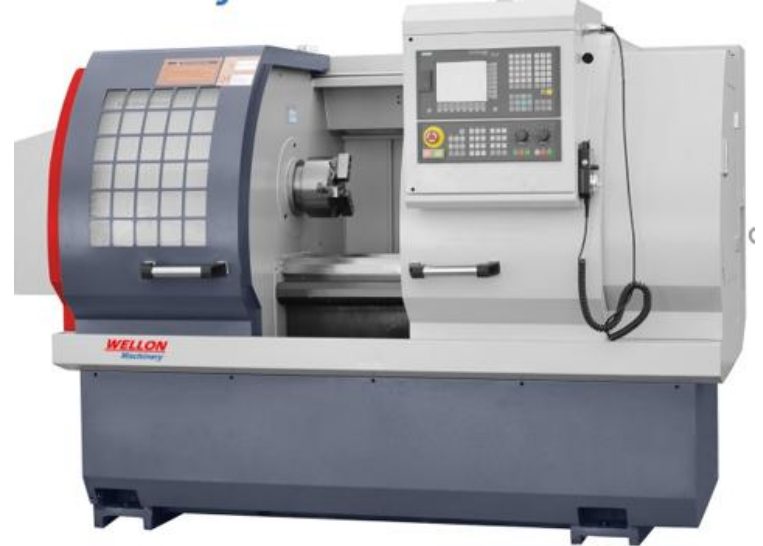

CNC Machine Mainly two types HMC and VMC Machine. HMC have a rotating workpiece and fix tool. And VMC have a rotating tool and workpiece is fix. In HMC Machine mostly round shape workpiece is place. In CNC Machine main operation like Facing, Groove, threading, drilling, knurling etc...

Revised Manuscript Received on April 11, 2020.

* Correspondence Author

Haresh Modhavadiya*, M.Tech.(CAD/CAM) Mechanical Engineering, Parul Institute of Technology, Parul University, Vadodara, India.E-mail: modhavadiyah22@gmail.com

Rakesh Prajapati, Mechanical engineering, Parul Institute of Technology, Parul University, Vadodara, India. E-mail: rakeshkumar.prajapati@paruluniversity.ac.in

Bhupesh Goyal, Mechanical engineering, Parul Institute of Technology, Parul University, Vadodara, India. E-mail: bhupesh.goyal@paruluniversity.ac.in

(C) The Authors. Published by Blue Eyes Intelligence Engineering and Sciences Publication (BEIESP). This is an open access article under the CC BY-NC-ND license (http://creativecommons.org/licenses/by-nc-nd/4.0/)

\section{WORKING PRINCIPLE}

CNC Machine works under close circle framework which implies when CNC read the Program and execute and criticism returning to the CNC Framework. By controlling the relative movements between the tool and work piece geometrical shapes are machined. Control the relative movements through coded letter numbers is known as numerical control of machine apparatuses.

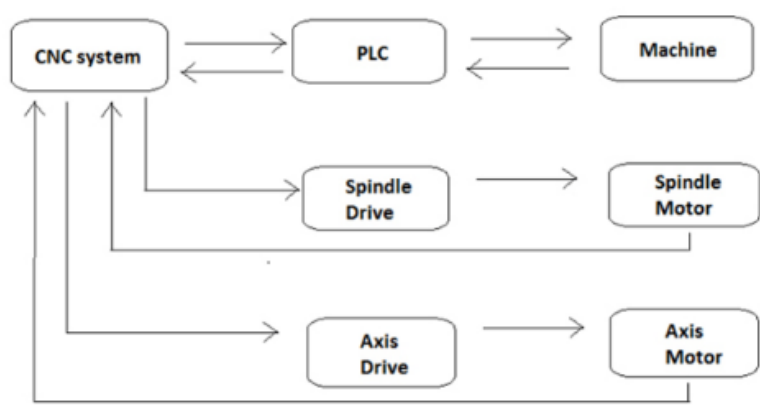

Fig.1 CNC Machine Working Principle

\section{LITERATURE REVIEW}

The review papers are mainly referring to reducing the weight of CNC Lathe bed with gray cast iron material and majority of the published work applies to them. At el. B.MALESWARA SWAMI[1]: In this paper the 3D model can be done by using CATIA software. For Analysis HYPERMESH software used.. Here material used CAST IRON G15, CAST IRON G40 AND CAST IRON G70. From the above material, the G15 chose as the best material because of low stress and high natural frequencies, At el. SUJIT GANESH KORE[2]: In this paper, a machine bed chose for the total analysis for static loads. The exploration work completed to decrease the heaviness of the machine bed, reduced the stress induced in the lathe bed and to lessen the relocation. In this work 3D model done by using SOLID EDGE V20 software. Analysis done by ANSYS 13 software. Material used CAST IRON, ST-STEEL and AL-ALLOY. The weight optimization of machine bed accomplished (Around 15\%) for all the three material, consequently the manufacturing cost additionally diminished. The von-misses stress for Upgraded model 2 and Upgraded model 3 diminished. (Approximately 19\%).At el. JUTURI SAIDAIAH [3]: structural and modal analysis completed on lathe bed at maximum load conditions.

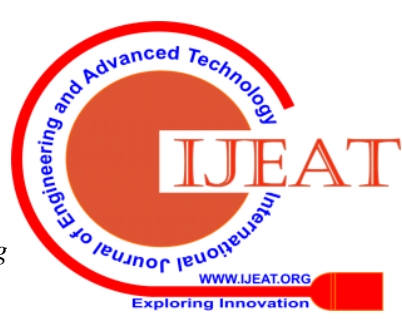


These simulation results used to decrease the heaviness of the lathe bed without breaking down its structural strength and damping limit by including ribs and expelling mass where less deformation and stresses induced. FEA analysis of modified lathe bed carried out with Gray cast iron and Epoxy-granite which is a mixture of granite and epoxy resin-hardener as an alternative material. Effectiveness of both materials compared in terms of induced stresses. Deformation and weight reduction. Lathe bed CAD models generated with CREO software. The analyses did utilizing ANSYS APDL software. The outcomes appeared as contour plots and tabulated, to analyses the impact of weight decrease on the structural integrity of the machine bed before and after the weight reduction and conclusions drawn about the optimized design. The current lathe bed weight is $105.816 \mathrm{Kg}$, after optimize the design weight of the bed has reduced to 97.992 $\mathrm{kg}$. Weight decrease is equivalent to $7.4 \%$ base model weight. Also Structural and modal analyses carried out for modified lathe bed with Epoxy granite material. By changing lathe bed material bed weight has decreased to $39.469 \mathrm{~kg}$ weight decrease is equivalent to $62.7 \%$ base model weight.

\section{PROBLEM STATEMENT}

MGX200 CNC Lathe bed weight was 1149 kg so company want to reduce the weight. This lathe bed is chosen for the complete analysis for both static and natural frequencies. At that point investigation is completed to diminish the heaviness of the machine bed without falling its structural rigidity and the accuracy of the machine tool by decreasing the material where lathe bed under goes less stress and deformation area likewise FE analysis will be done with grey cast iron material by applying on modified lathe bed.

\section{Mgx200 Cnc Machine Specification}

Table 1: MGX200 CNC Machine specification

\begin{tabular}{|c|c|c|}
\hline 1 & Max. Turning Diameter & $200 \mathrm{~mm}$ \\
\hline 2 & Max. Turning length & $170 \mathrm{~mm}$ \\
\hline \multirow[t]{2}{*}{3} & Swing Over bed & $380 \mathrm{~mm}$ \\
\hline & SPINDLE DRIVE & \\
\hline 4 & Spindle Motor Power & 7.5HP \\
\hline \multirow[t]{2}{*}{5} & Speed Range & $3000 \mathrm{rpm}$ \\
\hline & AXES SLIDES & \\
\hline 6 & X Axis Strokes & $350 \mathrm{~mm}$ \\
\hline 7 & Z Axis Strokes & $200 \mathrm{~mm}$ \\
\hline \multirow[t]{2}{*}{8} & Rapid Traverse: X\&Y Axis & $24 \mathrm{~m} / \mathrm{min}$. \\
\hline & MAIN SPINDLE & \\
\hline 9 & Spindle Nose & A2-6 \\
\hline 10 & Bore Through Spindle & $55 \mathrm{~mm}$ \\
\hline 11 & Bar Capacity & $42 \mathrm{~mm}$ \\
\hline \multirow[t]{2}{*}{12} & Chuck size & $200 \mathrm{~mm}$ \\
\hline & ACCURACY & \\
\hline 13 & Position Accuracy & $0.015 \mathrm{~mm}$ \\
\hline 14 & Repeatability & $\pm 0.003 \mathrm{~mm}$ \\
\hline
\end{tabular}

\section{MODELING AND ANALYSIS}

\section{Part Drawing of CNC Lathe Bed MGX200}
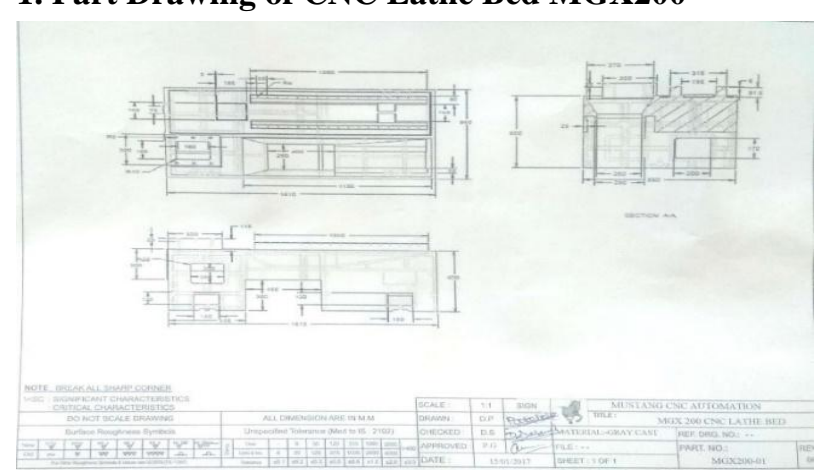

Fig.2. Detail Drawing of MGX200 CNC Lathe Bed

\section{Material:}

Gray Cast is amalgam of Carbon and silicon with iron. It contains 2.53 to $8 \% \mathrm{C}, 1.1-1.8 \% \mathrm{Si}, 0.4-1 \% \mathrm{MN}, 0.15 \% \mathrm{P}$ and $0.10 \%$ S. Graphite pieces possess about $10 \%$ of the metal volume.

It has high compressive strength, low tensile strength, high rigidity, high fluidity and ability to make sound casting. It has relatively low melting temperature $1130^{\circ}$ to $1250^{\circ} \mathrm{C}$. It easily machining.

Table 2: Material Property of gray cast iron

\begin{tabular}{|c|c|c|}
\hline Sr. No. & Properties & Values \\
\hline 1 & Density & $7.2 \mathrm{e}-006 \mathrm{~kg} \mathrm{~mm}^{-3}$ \\
\hline 2 & $\begin{array}{c}\text { Coefficient of Thermal } \\
\text { Expansion }\end{array}$ & $1.1 \mathrm{e}-005 \mathrm{C}^{-1}$ \\
\hline 3 & Specific Heat & $4.47 \mathrm{e}+005 \mathrm{~mJ} \mathrm{~kg}^{-1} \mathrm{C}^{-1}$ \\
\hline 4 & Thermal Conductivity & $5.2 \mathrm{e}-002 \mathrm{~W} \mathrm{~mm}^{-1} \mathrm{C}^{-1}$ \\
\hline 5 & Resistivity & $9.6 \mathrm{e}-005 \mathrm{ohm} \mathrm{mm}^{-1}$ \\
\hline 6 & Compressive Ultimate Strength & $820 \mathrm{Mpa}$ \\
\hline 7 & Tensile Ultimate Strength & $240 \mathrm{MPa}$ \\
\hline 8 & Young's Modulus & $1.1 \mathrm{e}+005 \mathrm{MPa}$ \\
\hline
\end{tabular}

\section{Modeling}

The CNC lathe bed created a 3D model in Autodesk Inventor Professional 2018 software.

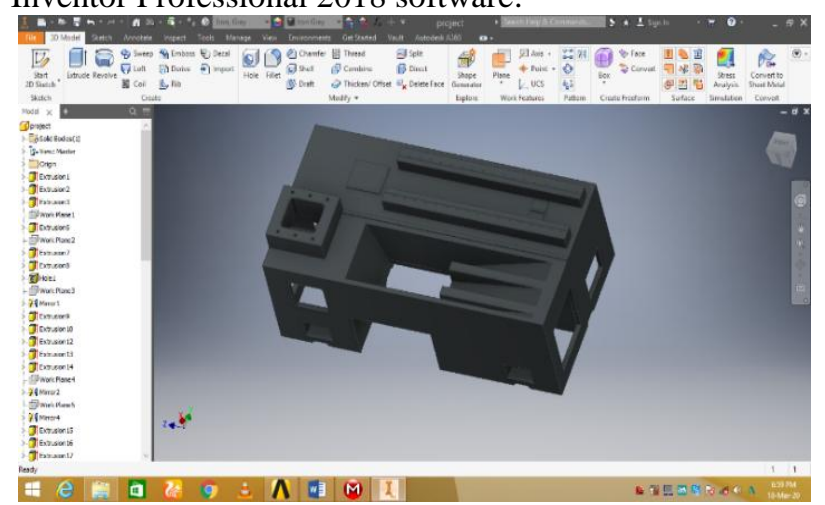

Fig.3. CNC Lathe Bed MGX200

\section{ANSYS 16.0 Software:}

\section{A.MGX200 CNC Lathe Bed Import:}

Here we can import MGX200 CNC Lathe bed model in ANSYS 16.0 Software

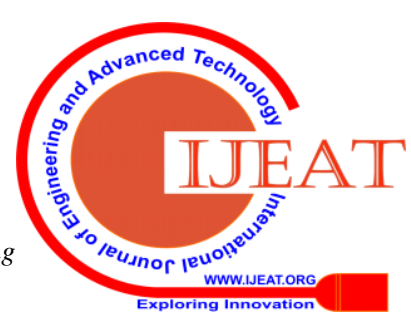




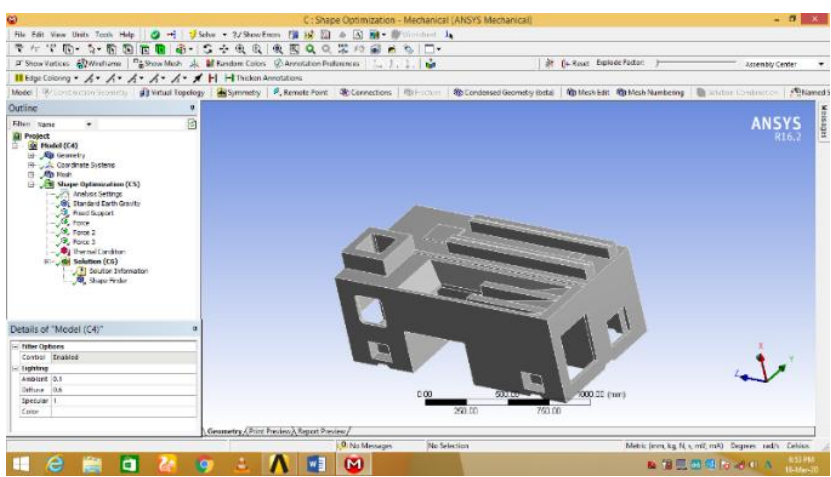

. Fig.4. CNC Lathe Bed Import in ANSYS 16.0

\section{B.Meshing:}

Here Hex Dominant method used for mesh. This meshing mostly used in the industries for the accurate mesh result. Meshing of solid model is $5.0 \mathrm{~mm}$, element edge length adjusted to $2.50 \mathrm{~mm}$ in order to obtain a regular uniform mesh.

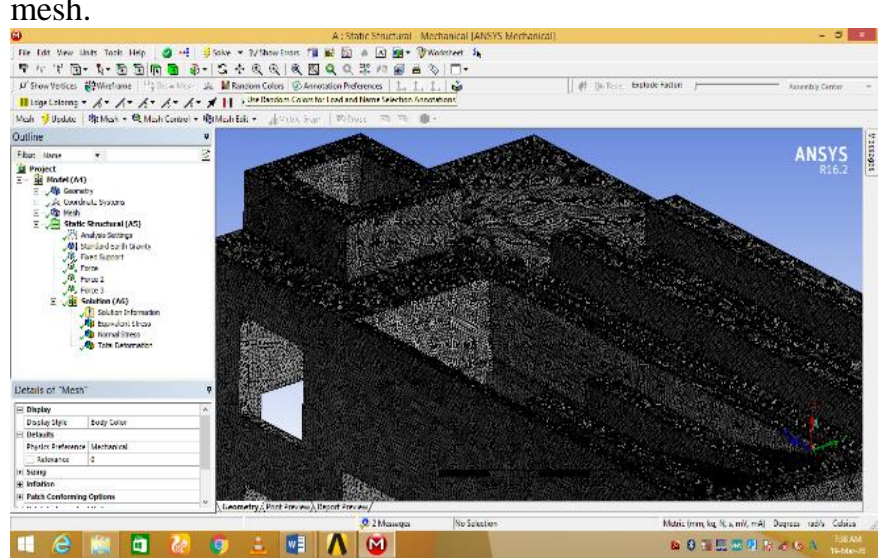

Fig.5. Applied Meshing on CNC Lathe Bed MGX200

\section{C.Boundary Condition:}

1) The determination of limit states of the CNC Machine bed base is fixed on the floor.

2) Gravitational force applied for stress distribution and deformation because of self-weight.

3) According to Industrial data $8000 \mathrm{~N}$ force applied on Head Stock.

4) According to Industrial data $6000 \mathrm{~N}$ force applied on Sliding portion.

5) According to Industrial data $2500 \mathrm{~N}$ force applied on Tail Stock.

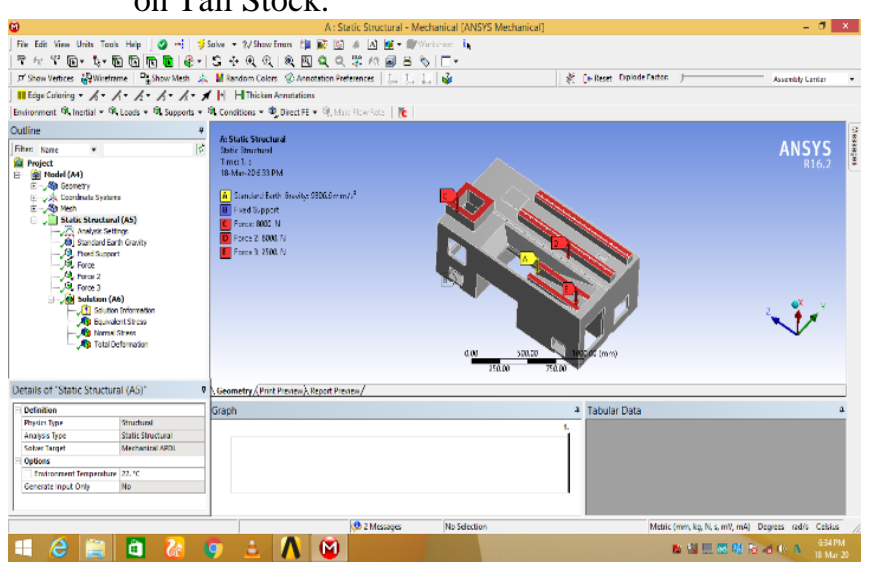

Fig.6. Applied Boundary Condition on MGX200 CNC Lathe Bed

\section{D.Static and Vibration Analysis of Existing Bed}
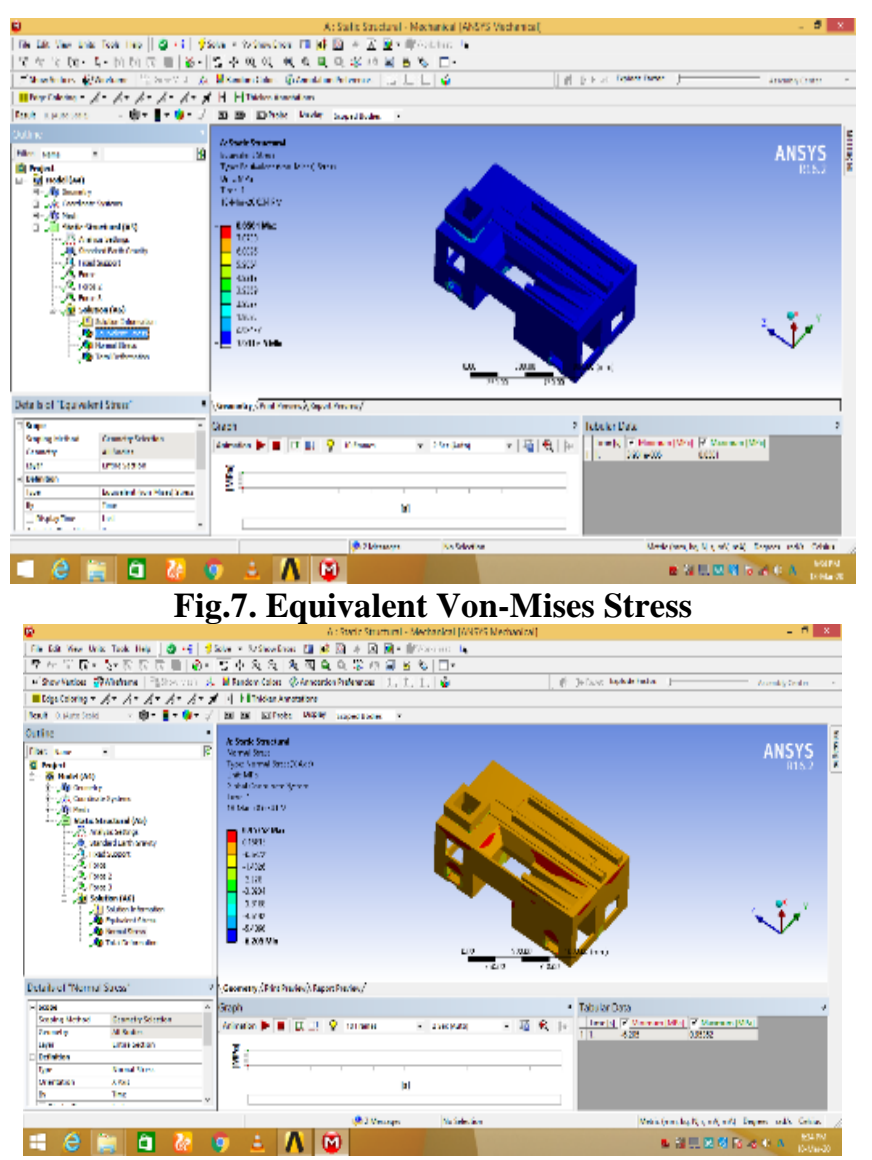

Fig.8. Normal Stress
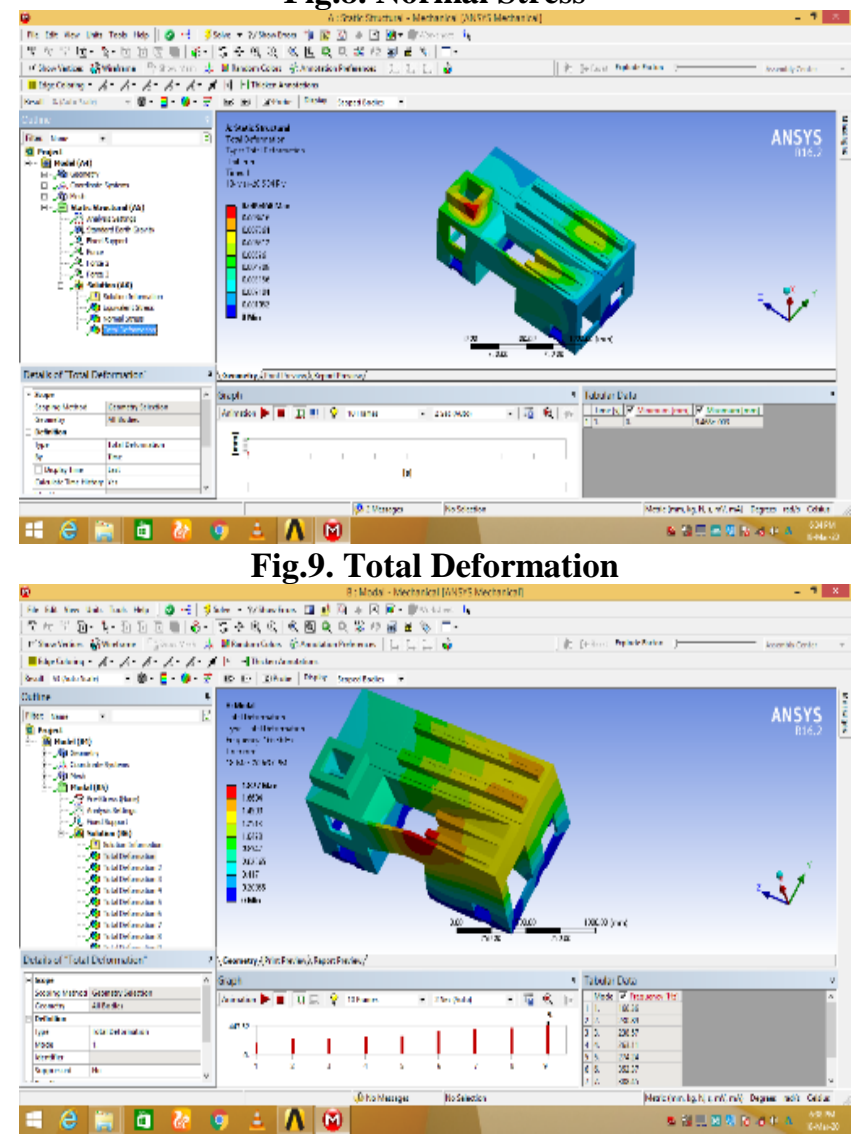

Fig.10. Natural Frequency Mode 1 Deformation

Published By:

Blue Eyes Intelligence Engineering \& Sciences Publication

(c) Copyright: All rights reserved.

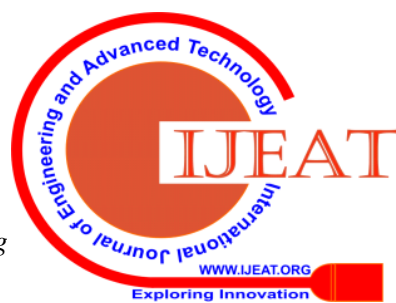




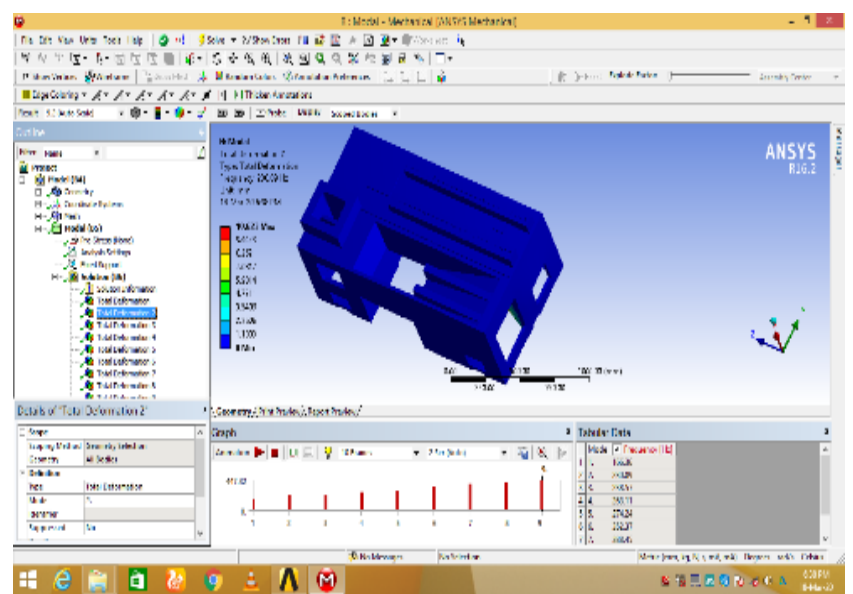

Fig.11. Natural Frequency Mode 2 Deformation

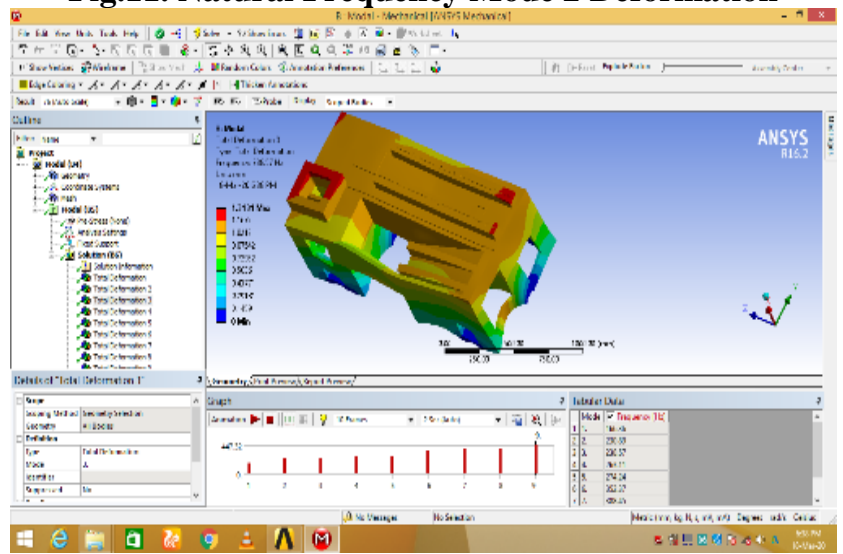

Fig.12. Natural Frequency Mode 3 Deformation

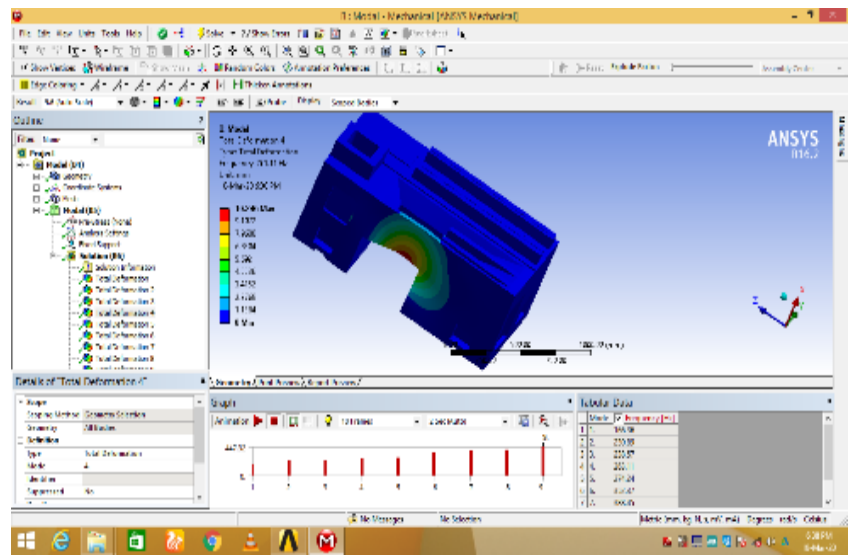

Fig.13. Natural Frequency Mode 4 Deformation

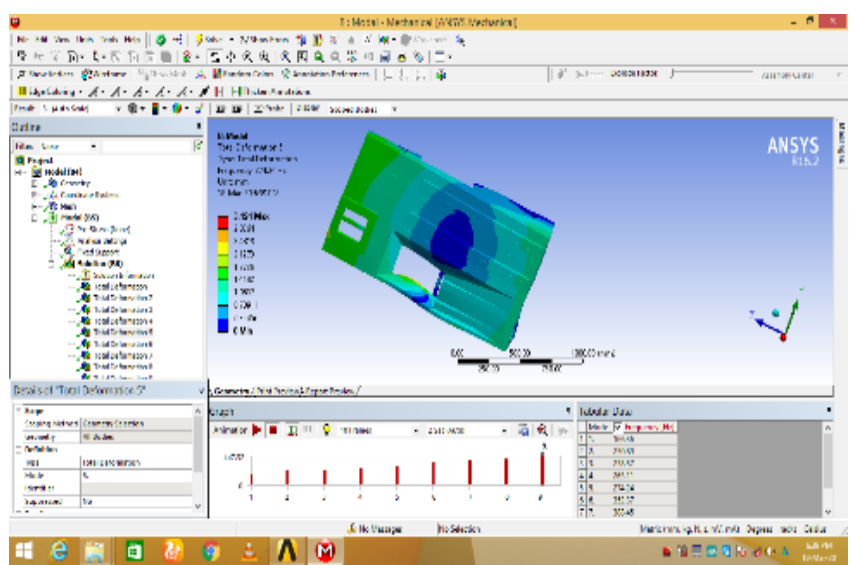

Fig.14. Natural Frequency Mode 5 Deformation

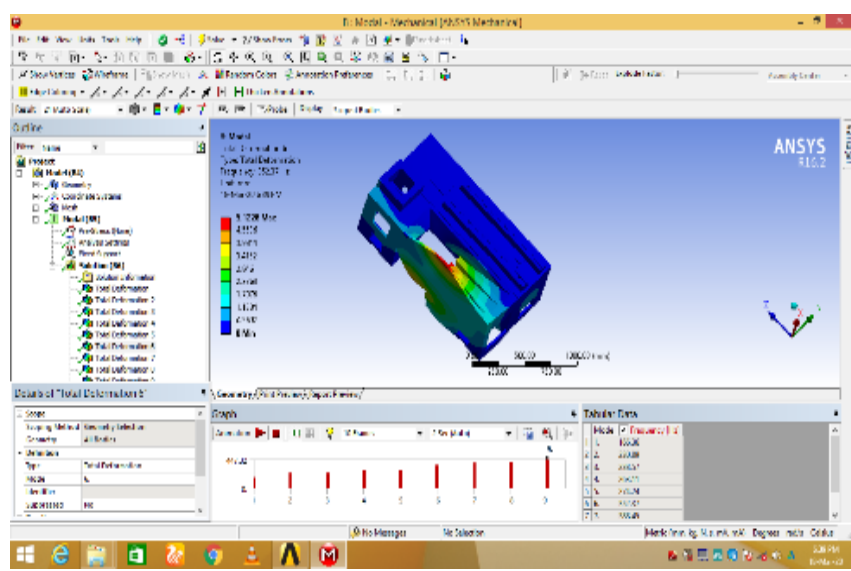

Fig.15. Natural Frequency Mode 6 Deformation

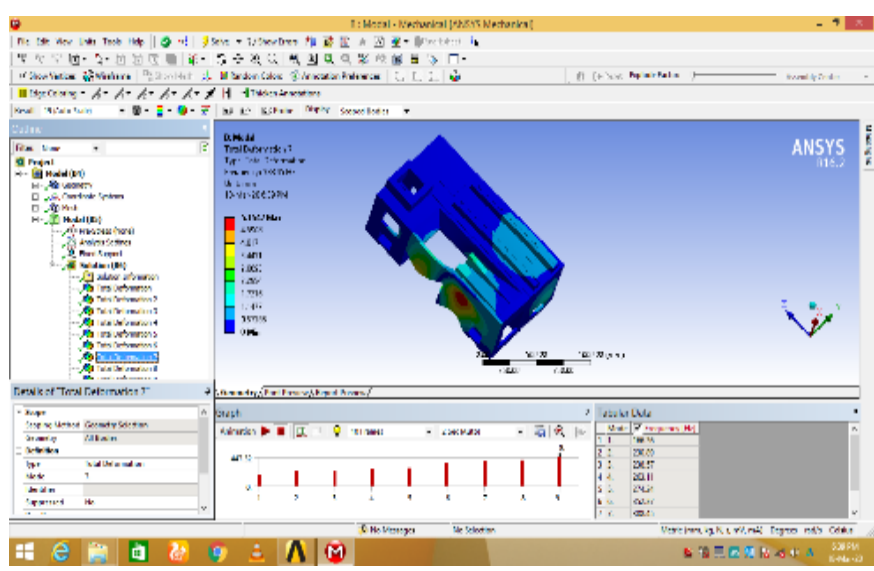

Fig.16. Natural Frequency Mode 7 Deformation

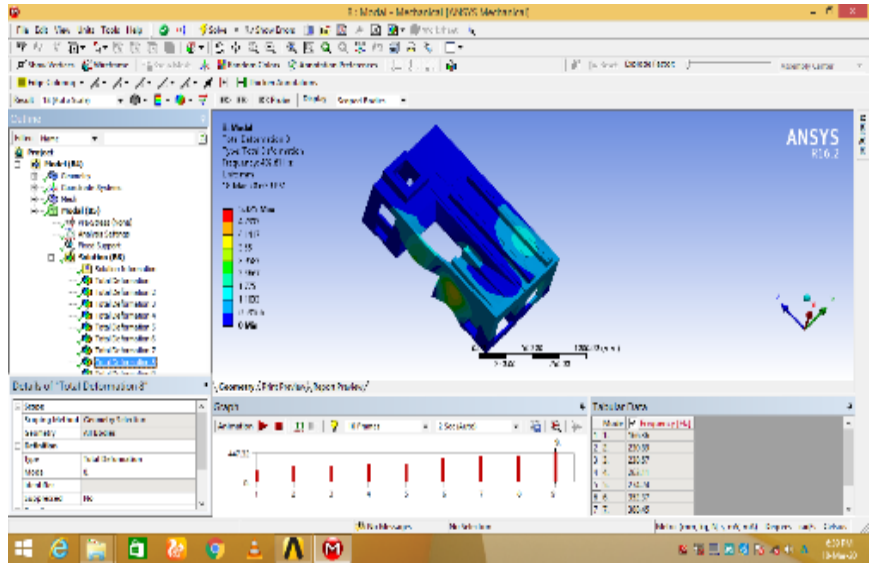

Fig.17. Natural Frequency Mode 8 Deformation

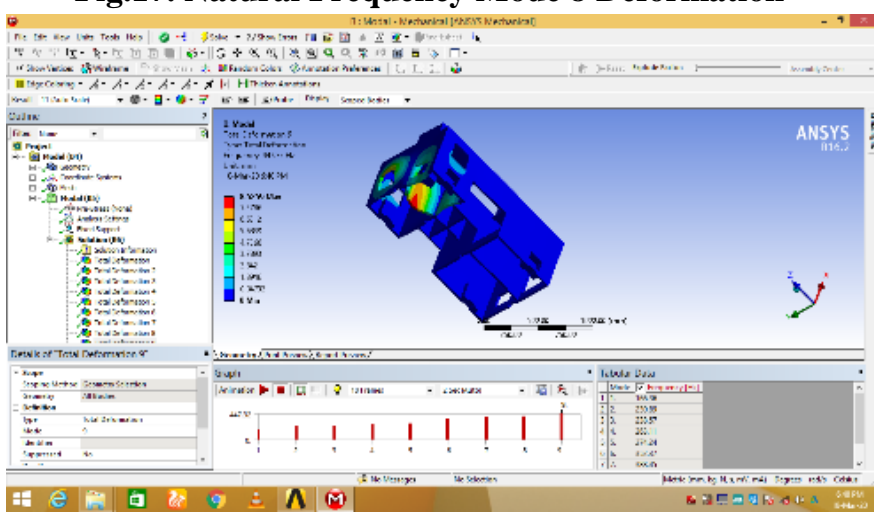

Fig.18. Natural Frequency Mode 9 Deformation

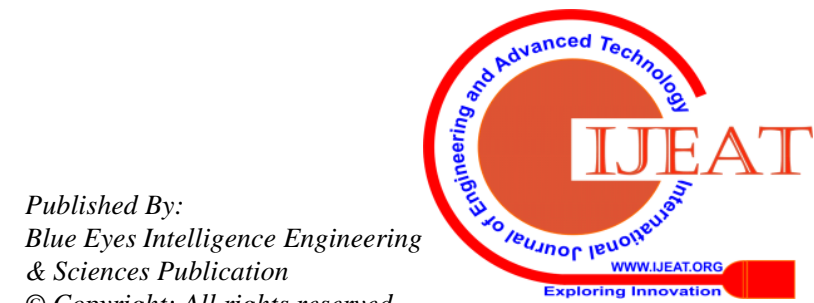

Retrieval Number: D7793049420/2020@BEIESP DOI: 10.35940/ijeat.D7793.049420

Journal Website: www.ijeat.org 
E.Static and Vibration Analysis After Modification of Bed:

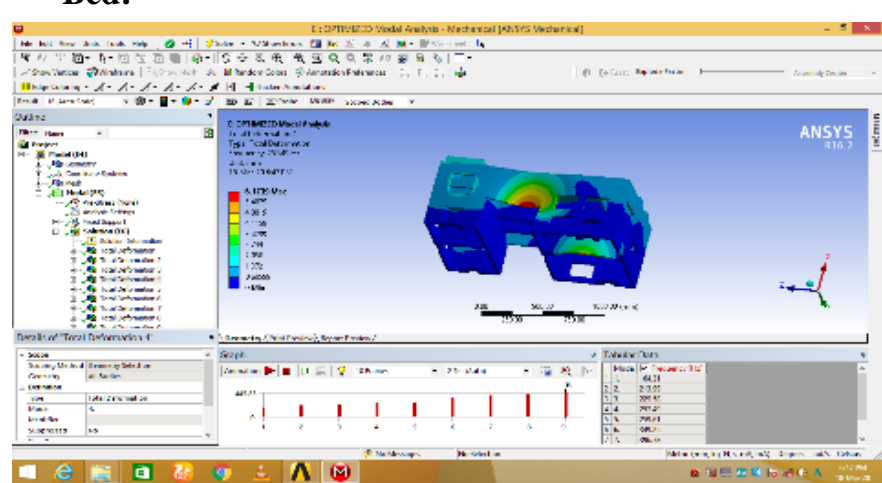

Fig.19. Equivalent Von-Mises stress

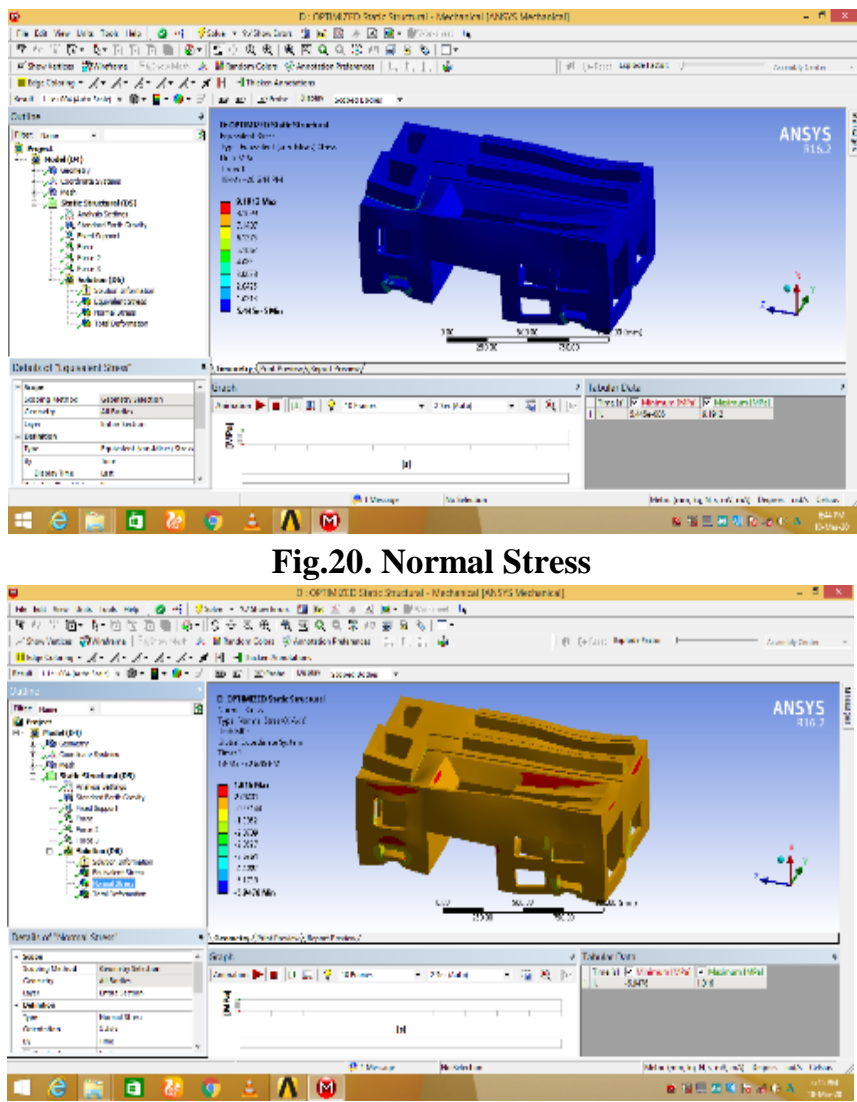

Fig.21. Total Deformation

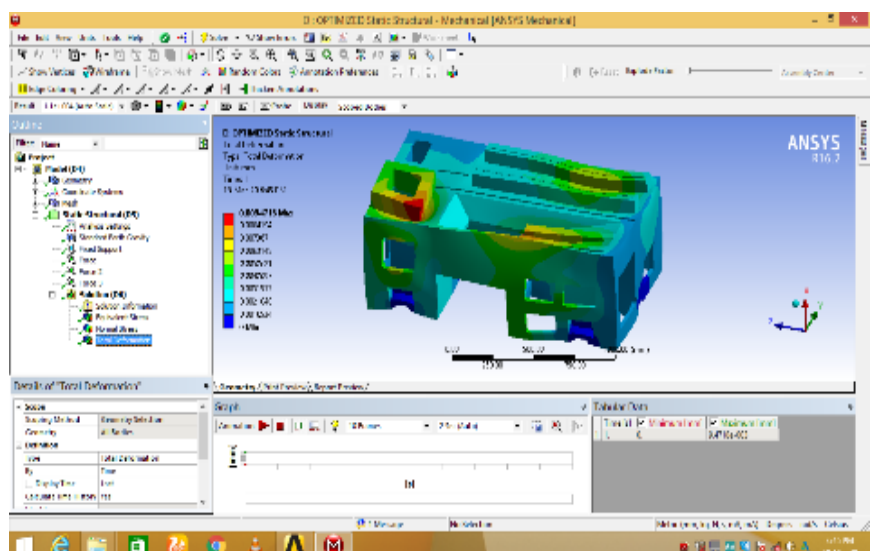

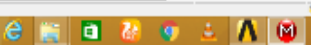

Fig.22. Natural Frequency Mode 1 Deformation

Retrieval Number: D7793049420/2020@BEIESP DOI: 10.35940/ijeat.D7793.049420

Journal Website: www.ijeat.org

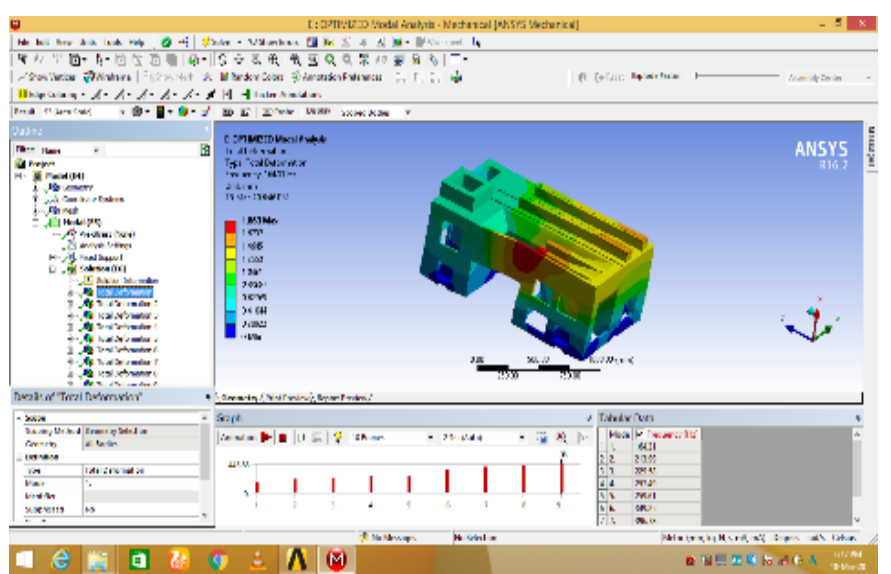

Fig. 23. Natural Frequency Mode 2 Deformation

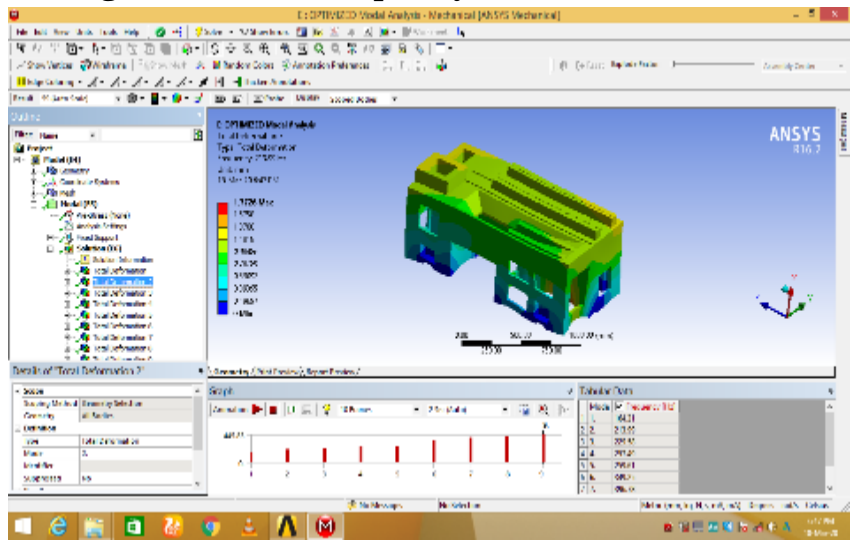

Fig.24. Natural Frequency Mode 3 Deformation

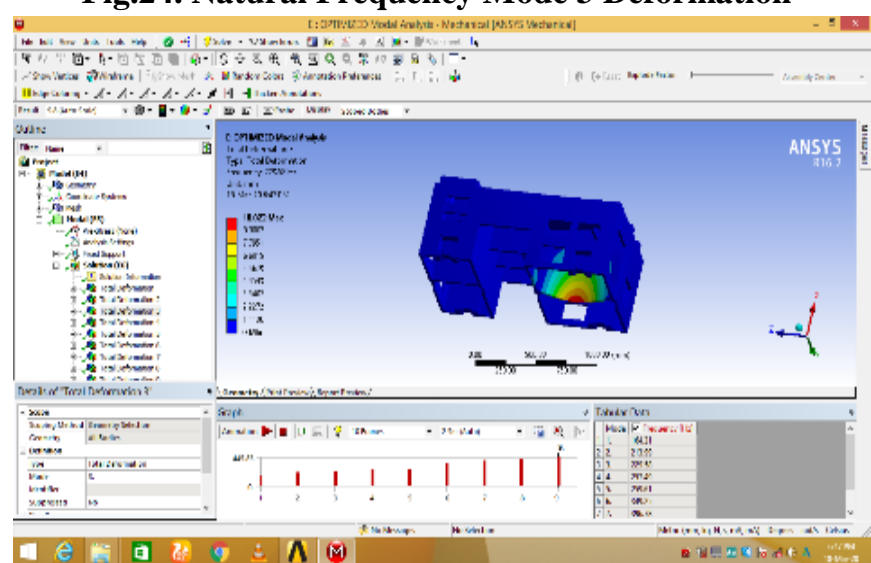

Fig.25. Natural Frequency Mode 4 Deformation

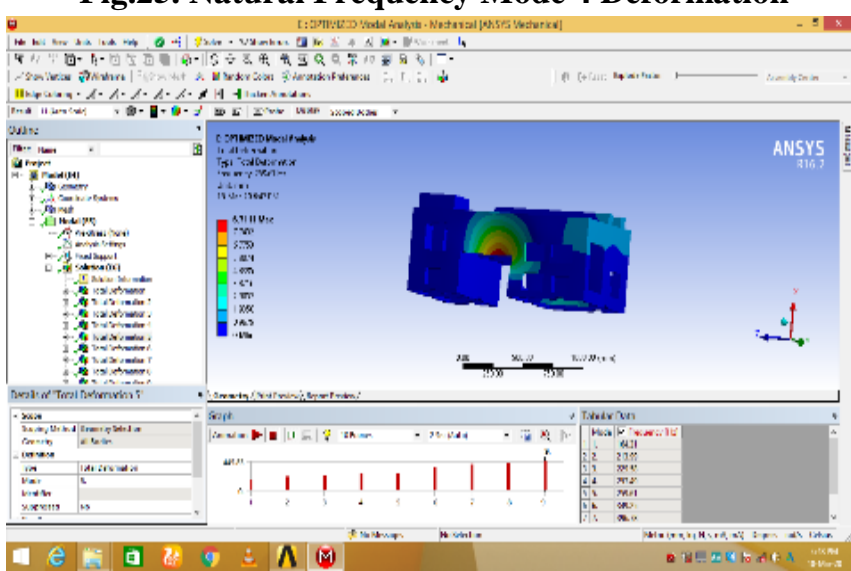

Fig.26. Natural Frequency Mode 5 Deformation

Blue Eyes Intelligence Engineering \& Sciences Publication

(c) Copyright: All rights reserved.

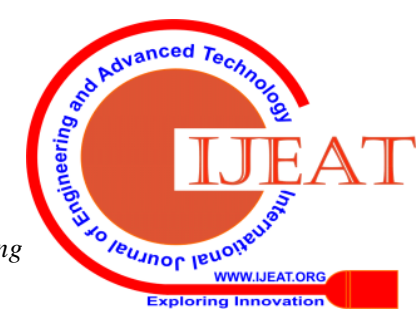




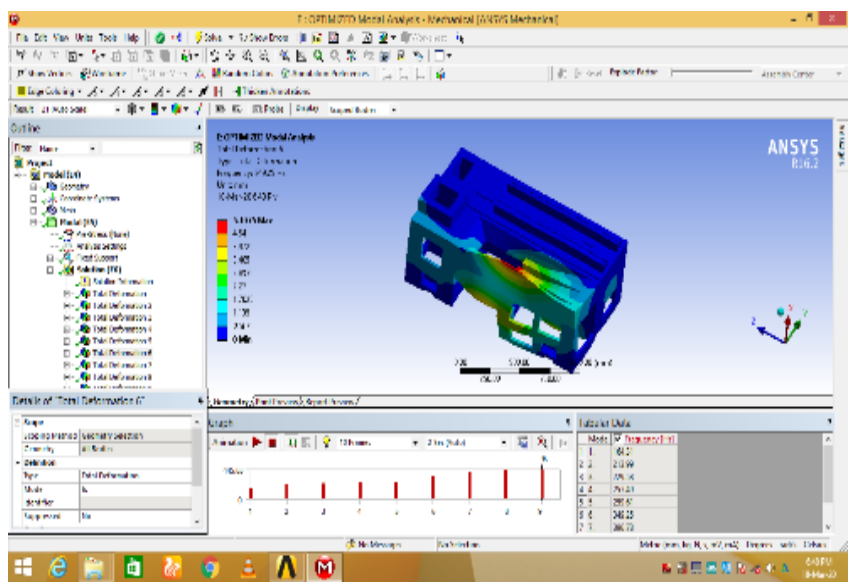

Fig. 27. Natural Frequency Mode 6 Deformation

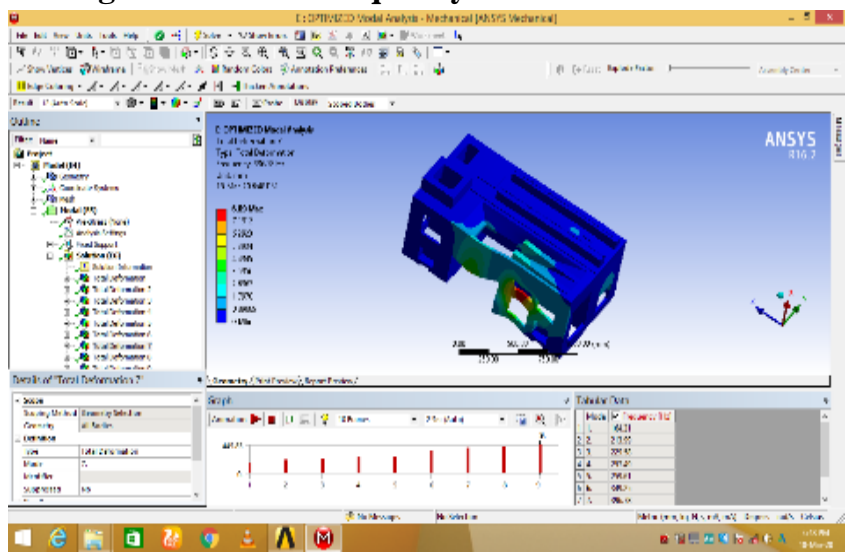

Fig.28. Natural Frequency Mode 7 Deformation

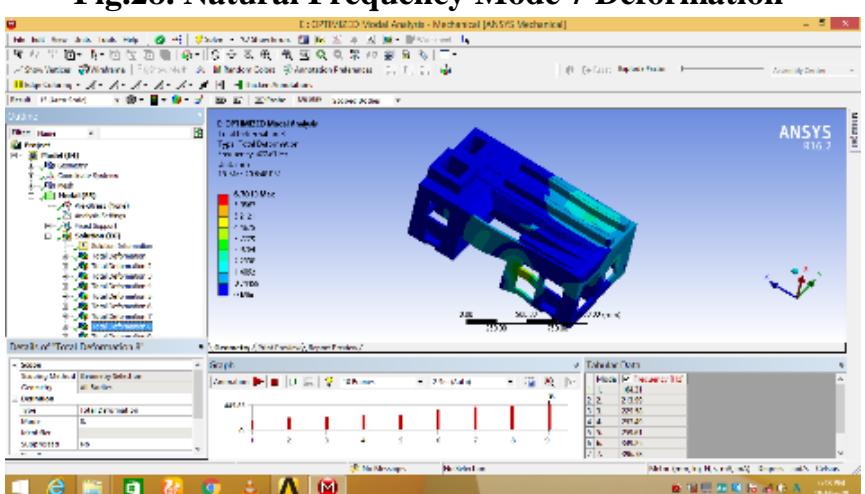

Fig.29. Natural Frequency Mode 8 Deformation

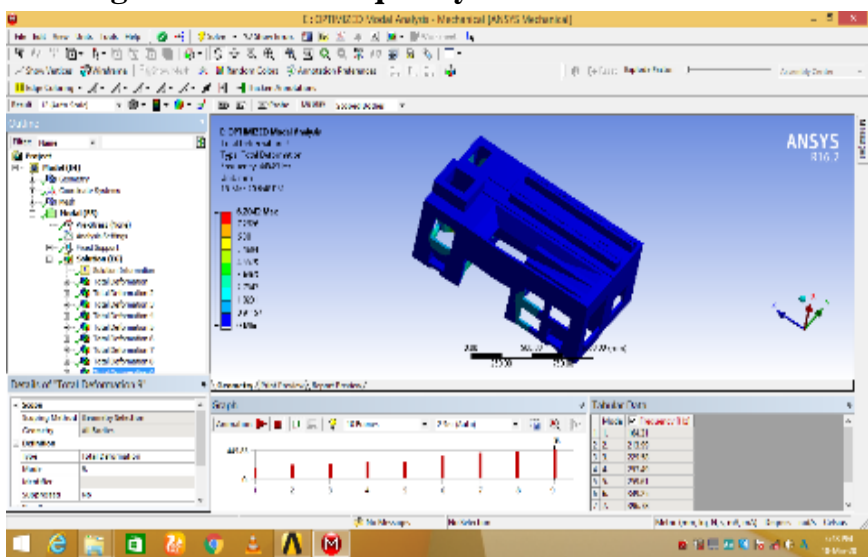

Fig.30. Natural Frequency Mode 9 Deformation

F. Result and Discussion:

TABLE 3: Comparison of Structural Analysis

\begin{tabular}{|c|c|c|c|c|c|}
\hline Parameter & $\begin{array}{c}\text { CNC Lathe } \\
\text { Bed } \\
\text { (Industrial } \\
\text { Data }\end{array}$ & $\begin{array}{c}\text { Existing CNC } \\
\text { Lathe Bed } \\
\text { ANSYS } \\
\text { Software Data }\end{array}$ & $\begin{array}{c}\text { Modified } \\
\text { CNC Lathe Bed } \\
\text { ANSYS } \\
\text { Software Data }\end{array}$ & $\begin{array}{c}\text { Modified CNC } \\
\text { Lathe Bed } \\
\text { CNC Lathe Bed With Gray } \\
\text { Cast Iron Grade 40 ANSYS } \\
\text { Software Data }\end{array}$ & $\begin{array}{c}\text { Modified } \\
\text { (Industrial Data }\end{array}$ \\
\hline $\begin{array}{c}\text { Normal Stress(X-Direction) } \\
\text { (Mpa) }\end{array}$ & 0.9838 & 0.95352 & 1.016 & 1.0558 \\
\hline $\begin{array}{c}\text { Equivalent(Von-Mises) } \\
\text { Stress (Mpa) }\end{array}$ & 8.9851 & 8.8581 & 9.1912 & 9.1124 \\
\hline Total Deformation (Mm) & 0.0091529 & 0.009468 & 0.0094718 & 9.1536 \\
\hline
\end{tabular}

TABLE 4: Comparison of Vibration Analysis

\begin{tabular}{|c|c|c|c|c|}
\hline \multicolumn{2}{|c|}{ Parameter } & Existing CNC Lathe & $\begin{array}{c}\text { Modified } \\
\text { CNC Lathe Bed }\end{array}$ & $\begin{array}{c}\text { Modified } \\
\text { CNC Lathe Bed With Gray Cast Iron Grade 40 }\end{array}$ \\
\hline Mode 1 & Frequency(Hz) & 166.36 & 164.31 & 169.35 \\
\hline Mode 2 & Frequency(Hz) & 230.89 & 213.99 & 220.65 \\
\hline Mode 3 & Frequency(Hz) & 238.57 & 229.58 & 236.99 \\
\hline Mode 4 & Frequency(Hz) & 263.11 & 257.49 & \\
\hline Mode 5 & Frequency(Hz) & 274.24 & 259.61 & 267.83 \\
\hline
\end{tabular}

Retrieval Number: D7793049420/2020@BEIESP DOI: 10.35940/ijeat.D7793.049420

Journal Website: www.ijeat.org

\section{Published By:}

Blue Eyes Intelligence Engineering \& Sciences Publication

(C) Convriaht: All riahts reserved.

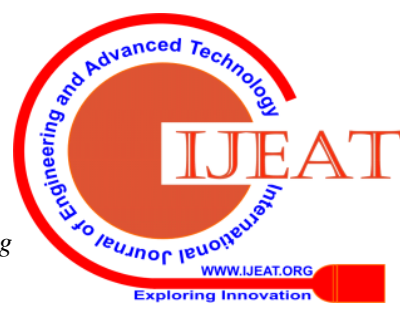




\begin{tabular}{|c|c|c|c|c|}
\hline Mode 6 & Frequency(Hz) & 352.37 & 349.78 & 360.31 \\
\hline Mode 7 & Frequency(Hz) & 388.45 & 396.78 & 409.39 \\
\hline Mode 8 & Frequency(Hz) & 402.61 & 422.61 & 460.42 \\
\hline Mode 9 & Frequency(Hz) & 447.32 & 445.83 & \\
\hline
\end{tabular}

- The result of the Static Structure parameter is almost the same as an existing and modified bed.

- We tried to change the material grade but Static Structure and Vibration Parameter is almost the same as an existing material.

\section{CONCLUSION}

- The weight of the CNC lathe bed before modification is $1149 \mathrm{Kg}$, Subsequent to changing the design weight of the bed has decreased to $1115 \mathrm{~kg}$. This weight decrease is equivalent to $2.95 \%$ base model weight.

- We get practically same natural frequencies in case of modified model.

\section{REFERENCES}

1. Hongping Yang, "Static and dynamic characteristics modeling for CK61125 CNC lathe bed basing on FEM". GCMM, ISSN NO. 489-496, year 2016.

2. B. Malleswara Swami1 "Design and Structural Analysis of CNC Vertical Milling Machine Bed” IJAET, ISSUE NO.-0976-3945, DEC. 2012

3. Juturi Saidaiah "Weight Optimization of Lathe Bed by Design Modification and Epoxy Granite", SSRG-IJME, ISSN NO. 2348-83607 July 2017

4. S. Syath Abuthakeer "Structural Redesigning of a CNC Lathe Bed to Improve Its Static and Dynamic Characteristics" NSPGS, ISSN-1584-2673, Year 2011

5. C.C. Hong "Static Structure Analysis of Great Five Axis Turning Milling Complex CNC Machine" YEAR 2016.

6. Gururaj Deshpande, "Structural Analysis for Weight Optimization of Machine Tool Bed” IJIERT, ISSN NO. 2394-3696, YEAR 2016.

7. Ashish Shrivastav, "Optimization of Design Parameter of Vertical Machining Center Column for The Weight and Rigidity", IJAERO, ISSN NO. 2348-6406, MARCH,2017.

8. Ripalkumar C. Patel "Finite Element Analysis and Optimization of Conventional Lathe Bed Using CAE Tool” ISBN-978-81-929339-1-7, APRIL 2015

9. Mr. B. Sakthi "Structural Redesigning of A CNC Lathe Bed to Improve Its Stiffness and Damping Characteristics" AFEH-IJE, ISSN 1584-2673, YEAR 2011.

10. Sujeet Ganesh Kore "Design and Analysis of a Machine Tool Structural Bionics IONICS” IJMERR, ISSN-2278-0149, JULY 2014.

11. Myo Min Zaw "Strength Analysis of Bed ways for CNC Lathe Machine" GMSARN International Conference on Sustainable Development: Issue and Prospects for the GMS 12-14 Nov. (2008)

12. Bernard Budiansky, "On the minimum weights of compression structures", in International Journal of Solids and Structures, volume 36, (1999), pp. 3677- 3708.

13. Kim D I., Jung S C, Lee J E. Parametric study on design of composite-foam-resin concrete sandwich structures for precision machine tool structures. Composite Structures, (2006), 75, 408-414.

14. TAPAN A PANDYA, M. Tech, "Evaluation of alternative material for lathe bed" May 2016.

15. Damping characteristics of composite hybrid spindle covers for high speed machine tools Jung Do Suha, Seung Hwan Changa, Dai Gil Leea, Jin Kyung Choib and Bo Seon Parkc, Journal of Materials Processing Technology, Volume 113, Issues 1-3, 15 June (2001), Pages 178-183.

Engineering in Mechanical Engineering from V.V.P. ENGG. College, Rajkot. Under Gujarat Technical University. M.Tech. CAD/CAM is pursuing in Parul University.

Prof. Rakesh Prajapati is currently working as an Assistant professor at Parul Institute of Technology, Parul University. He did his mechanical engineering M. Tech CAD/CAM from Charusat University, Changa. His research area is CNC Machine, CNT, Composite, Additive Manufacturing.

Prof. Bhupesh Goyal is currently working as an Assistant professor at Parul Institute of Technology, Parul University. He did his mechanical engineering M. Tech CAD/CAM from M. S. University. His research area is CNC Machine, 3D Printing, Metal Forming, Composite. 